\title{
Dark Matter and Galaxy Formation
}

\author{
K. C. Freeman \\ Mount Stromlo and Siding Spring Observatories, Australian National University, \\ Private Bag, Weston Creek PO, ACT 2611, Australia \\ kcf@mso.anu.edu.au \\ Received 1996 October 29, accepted 1996 November 28
}

\begin{abstract}
In addition to large numbers of normal galaxies, the multibeam $\mathrm{H}_{\mathrm{I}}$ Parkes all-sky survey will probably discover intergalactic $\mathrm{H}$ I clouds, very low-surface-brightness, gas-rich galaxies, and small $\mathrm{H}_{\mathrm{r}}$ clouds in nearby groups of galaxies. I will briefly discuss some aspects of why these classes of objects are interesting in the context of dark matter and galaxy formation.
\end{abstract}

Keywords: dark matter — low-surface-brightness galaxies - intergalactic clouds groups of galaxies - galaxy formation

\section{Detection Limits}

I consider first some estimates of the likely detection limits for the multibeam system. If we take the present Parkes $\mathrm{H}$ I system as a guide to the sensitivity of the multibeam system, the empirical detection limit on the integrated $\mathrm{H}_{\mathrm{I}}$ emission from an unresolved galaxy (from my own experience) is about

$$
1 \cdot 5\left(\frac{\Delta V}{200}\right)^{\frac{1}{2}}\left(\frac{20}{t}\right)^{\frac{1}{2}} \mathrm{Jy} \mathrm{km} \mathrm{s}^{-1}
$$

where $\Delta V$ is the velocity width of the $\mathrm{H}_{\mathrm{I}}$ distribution in $\mathrm{km} \mathrm{s}^{-1}$ and $t$ is the integration time in minutes. This corresponds to an $\mathrm{H}$ I mass of

$$
M_{\mathrm{HI}}=3.5 \times 10^{5} d^{2}\left(\frac{\Delta V}{200}\right)^{\frac{1}{2}}\left(\frac{20}{t}\right)^{\frac{1}{2}} M_{\odot}
$$

where $d$ is the galaxy's distance in Mpc. For example:

- small $\mathrm{H}$ I objects in the nearest group of galaxies: $d=2.5 \mathrm{Mpc}, \Delta V=20 \mathrm{~km} \mathrm{~s}^{-1}, t=5 \mathrm{~min}$, and the detection limit is $M_{\mathrm{HI}}=1.4 \times 10^{6} M_{\odot}$.

- large objects at the redshift limit of the survey (about 14,000 $\mathrm{km} \mathrm{s}^{-1}$ ): $\quad d=200 \mathrm{Mpc}, \Delta V=$ $400 \mathrm{~km} \mathrm{~s}^{-1}, t=5 \mathrm{~min}, M_{\mathrm{HI}}=4 \times 10^{10} M_{\odot}$.

The Parkes beam diameter is $4.4 d(\mathrm{Mpc}) \mathrm{kpc}$ (e.g. $200 \mathrm{kpc}$ at a redshift of about $3000 \mathrm{~km} \mathrm{~s}^{-1}$ ), so galaxy-sized objects will be unresolved over most of the redshift range for the multibeam survey. This is also the case for the known intergalactic clouds, which are described briefly in the next section.

\section{Intergalactic Clouds}

The intergalactic $\mathrm{H}_{\mathrm{I}}$ clouds discovered so far are associated with individual or interacting galaxies, groups and clusters. They all have roughly similar sizes and $\mathrm{H}_{\mathrm{I}}$ masses. Here are some examples:

- The Leo group ring (Schneider 1989). Its diameter $D$ is about $200 \mathrm{kpc}$ and its $\mathrm{H}$ I mass $M_{\mathrm{HI}}$ is $1.7 \times 10^{9}$ $M_{\odot}$.

- The Virgo cloud (Giovanelli \& Haynes 1989), with $D=200 \mathrm{kpc}$ and $M_{\mathrm{HI}}=4 \times 10^{9} M_{\odot}$.

- The A2634 cloud (Giovanelli et al. 1995). Its linear dimensions are about $150 \times 100 \mathrm{kpc}$, and its $M_{\mathrm{HI}}=6 \times 10^{9} M_{\odot}$.

- The cloud discovered recently near the NGC 3256/3263 system by English (1994), with $D=120 \mathrm{kpc}$ and $M_{\mathrm{HI}}=6 \times 10^{9} M_{\odot}$.

The nature and astrophysical significance of these clouds is not yet clear. At least in some cases, like the NGC 3256/3263 example, it seems likely that they come from tidal interaction of galaxies within their parent system. Intergalactic clouds like these will not be detectable with 5 minute integrations much beyond a redshift of about $7000 \mathrm{~km} \mathrm{~s}^{-1}$.

\section{Low-surface-brightness Gas-rich Galaxies}

Low-surface-brightness (LSB) $\mathrm{H}$ I-rich galaxies will probably be discovered in significant numbers in the multibeam survey. I believe that these gas-rich LSB galaxies are astrophysically very interesting and, in this section, I will attempt to intepret them in terms of an argument due to Fall (1985).

\subsection{Collapse of a Disk within a Dark Corona}

In simulations of the growth of density fluctuations in the expanding universe (e.g. Zurek et al. 1988), blobs of matter acquire angular momentum from each other by tidal torques. Say a blob of matter (dark + gas) has a mass $M$, energy $E$ and angular momentum $J$. The parameter $\lambda=J|E|^{\frac{1}{2}} G^{-1} M^{-\frac{5}{2}}$ is dimensionless and is a measure of the ratio (rotational velocity)/(virial velocity) for the blob. 
For disks in centrifugal equilibrium, $\lambda \approx 0.45$. These cosmological simulations give mean values of $\lambda=0.05 \pm 0.03(\sigma)$ for identifiable galaxy-sized objects, almost independent of the mass of the blob, its density, initial overdensity, and the adopted fluctuation spectrum.

In a simplistic version of the present view of disk galaxy formation, the gas in the blob settles by dissipation into centrifugal equilibrium within the dark matter distribution. Let the gas and the dark matter be initially well mixed and have similar specific angular momentum $J / M$. Then the gas needs to dissipate (conserving its $J$ ) to form an equilibrium disk with $\lambda \approx 0 \cdot 45$, having started with $\lambda \approx 0.05$. We can estimate the collapse factor in terms of the initial $\lambda$. Say the dark matter has an $r^{-2}$ density distribution, truncated at some radius $r=r_{\mathrm{t}}$, and let its constant circular velocity be $V$. From the virial theorem, it follows that the halo has $(J / M)_{\mathrm{H}}=\sqrt{2} \lambda V r_{\mathrm{t}}$. Now assume that the gas settles to an exponential disk with scale length $h_{\mathrm{R}}$, in centrifugal equilibrium in the potential of the halo, so the disk has $(J / M)_{\mathrm{D}}=2 V h_{\mathrm{R}}$. Then if $(J / M)_{\mathrm{D}}=(J / M)_{\mathrm{H}}$, it follows that the collapse factor is

$$
r_{\mathrm{t}} / h_{\mathrm{R}}=\sqrt{2} / \lambda \approx 30
$$

For our Galaxy, $h_{\mathrm{R}} \approx 4 \mathrm{kpc}$ (e.g. Freeman 1987), so the truncation radius $r_{\mathrm{t}}$ of the halo should be about $120 \mathrm{kpc}$ : this is entirely consistent with the observed extent of the galactic dark halo (e.g. Freeman 1996).

\subsection{High- $\lambda$ Galaxies}

The observed distribution of the $\lambda$ values in simulations has a mean of about 0.05 with a tail extending up to about $\lambda=0.15$ (e.g. Zurek et al. 1988). From equation (3), the collapse factor is $\propto \lambda^{-1}$, so the mean surface density of the gas in the equilibrium disk is $\propto \lambda^{-2}$. A typical galactic disk now has a surface density $\Sigma \approx 100$ $M_{\odot} \mathrm{pc}^{-2}$ at $r=h_{\mathrm{R}}$, so a high- $\lambda$ disk with say $\lambda=0.15$ would have a much lower surface density, $\Sigma \approx 10 M_{\odot} \mathrm{pc}^{-2}$. The threshold surface density for massive star formation is believed to be about 5 to $10 M_{\odot} \mathrm{pc}^{-2}$ (e.g. Kennicutt 1989), so one might expect high- $\lambda$ galaxies to have low surface density, with star formation suppressed and confined to the higher-density inner regions. The typical $\mathrm{H}_{\mathrm{I}}$ surface densities in gas-rich LSB galaxies (giants and dwarfs) are in the range $2-5 M_{\odot} \mathrm{pc}^{-2}$.

\subsection{Some Low-surface-brightness HI-rich Galaxies}

Here are some examples of LSB HI-rich galaxies, both giants and dwarfs. Table 1 gives dynamical and structural parameters (radial velocity in $\mathrm{km} \mathrm{s}^{-1}$ or distance in Mpc estimated from the brightness of resolved objects in the nearby dwarfs NGC 2915 and DDO 154; central surface brightness in B mag $\operatorname{arcsec}^{-2}$; $\mathrm{H}_{\mathrm{I}}$ line width in $\mathrm{km} \mathrm{s}^{-1}$; absolute magnitude; exponential scale length in kpc for the luminous component). NGC 5084 is a recent addition to the list of likely LSB gas-rich giants. It is an edge-on galaxy with a faint, extended disk and a very large $\mathrm{Hr}$ line width. Its disk would be about 3 mag fainter in surface brightness if it were seen face-on, and would probably be undetected. We note that:

- all of these galaxies were discovered optically, by their brighter central regions

- the Tully-Fisher law breaks down for these systems, as one might expect

- the two dwarfs have very large $\mathrm{H}$ I diameters: their ratios $\left(\mathrm{H}_{\mathrm{I}}\right.$ diameter $) /($ Holmberg diameter $) \approx 5$.

Table 1. Dynamical and structural parameters for LSB galaxies

\begin{tabular}{lccccc}
\hline & $V_{0}$ & $B(0)_{\mathrm{c}}$ & $W_{\mathrm{O}}$ & $M_{\mathrm{B}}$ & $h_{\mathrm{R}}$ \\
\hline Malin 1 & 24750 & 26 & 455 & $-21 \cdot 0$ & 55 \\
F568-6 & 13830 & $23 \cdot 4$ & 674 & $-21 \cdot 2$ & 16 \\
1226+0105 & 23655 & $23 \cdot 3$ & 405 & $-21 \cdot 6$ & 12 \\
NGC 5084 & 1550 & 700 & & $-20 \cdot 8$ & \\
& & & & & \\
NGC 2915 & $5 \mathrm{Mpc}$ & $22 \cdot 4$ & 170 & $-15 \cdot 9$ & $0 \cdot 7$ \\
DDO 154 & $4 \mathrm{Mpc}$ & $23 \cdot 2$ & 110 & $-13 \cdot 8$ & $0 \cdot 4$ \\
\hline
\end{tabular}

Table 2 gives the integrated mass and blue $M / L$ ratio out to the outermost rotation point, and the total $\mathrm{HI}_{\mathrm{I}}$ mass and $\mathrm{HI}_{\mathrm{I}}$ mass-to-light ratio (all in solar units). We note that:

- the total $M / L$ ratios for these systems are much larger than the usual $M / L=3-5$ for spiral disks. Although their disks LSB may give them a fragile appearance, they are in fact no more fragile than other disk galaxies, because the dark matter dominates in their outer regions.

- the giant LSB gas-rich galaxies are detectable with the multibeam system out to redshifts of 7000 to $14000 \mathrm{~km} \mathrm{~s}^{-1}$ with 5 minute integrations.

- dwarf systems like NGC 2915 are detectable out to about $3000 \mathrm{kms}^{-1}$ in 5 minutes.

Table 2. Masses and $M / L$ ratios for LSB galaxies

\begin{tabular}{lcccc}
\hline & $M_{\text {tot }}$ & $M_{\text {tot }} / L$ & $M_{\mathrm{H} \mathrm{I}}$ & $M_{\mathrm{H} \mathrm{I}} / L$ \\
\hline Malin 1 & $2 \times 10^{12}$ & 55 & $1 \times 10^{11}$ & 3 \\
F568-6 & $9 \times 10^{11}$ & 20 & $2 \times 10^{10}$ & $0 \cdot 5$ \\
1226+0105 & $2 \times 10^{12}$ & 30 & $2 \times 10^{10}$ & $0 \cdot 4$ \\
NGC 5084 & $1.3 \times 10^{12}$ & 45 & $9 \times 10^{9}$ & $0 \cdot 3$ \\
NGC 2915 & $3 \times 10^{10}$ & 75 & $1 \times 10^{9}$ & 3 \\
DDO 154 & $4 \times 10^{9}$ & 75 & $3 \times 10^{8}$ & 6 \\
\hline
\end{tabular}

Briggs (1990) has argued that there could be $\sim 1000$ giant LSB galaxies with $M_{\mathrm{HI}}>10^{11} M_{\odot}$ nearer than the prototype object Malin 1. Although the LSB galaxies in Tables 1 and 2 were discovered 
optically because they have bright central regions, it is possible that there are undiscovered giant LSB galaxies which are dark or almost dark. If they exist and contain $\mathrm{HI}$ in masses similar to the giants listed in Tables 1 and 2, then the multibeam survey should detect them.

\section{Small H I Clouds in Nearby Groups}

Here I am considering nearby groups like the Sculptor group $(2.5 \mathrm{Mpc})$ and the Centaurus A group (3.5 $\mathrm{Mpc})$. These groups are dynamically unevolved and may be coming together for the first time. Table 3 gives the mean radius $\langle r\rangle$ in $\mathrm{Mpc}$ and the mean absolute peculiar velocity $\langle|V|\rangle$ in $\mathrm{km} \mathrm{s}^{-1}$ of the galaxies in each group, and the crossing time $t_{\text {cross }}$ in years. This compilation includes the bright galaxies and the many dwarf galaxies discovered in each group by Côté (1995).

Table 3. Dynamical parameters for nearby groups

\begin{tabular}{lccc}
\hline & $\langle r\rangle$ & $\langle|V|\rangle$ & $t_{\text {cross }}$ \\
\hline Scl & 0.66 & 127 & $4 \times 10^{9}$ \\
Cen A & 0.72 & 100 & $5 \times 10^{9}$ \\
\hline
\end{tabular}

The interest of groups like Scl and Cen A is that, in the usual picture of galaxy formation by secondary infall, the smallest mass elements are expected to have masses of about the Jeans mass at recombination $\left(\sim 10^{6} M_{\odot}\right)$. These are the basic building blocks of galaxies and are likely to be made of gas and dark matter. It seems possible that dynamically unevolved systems like these two groups, with their long crossing times, could contain significant numbers of these small primordial mass elements which have so far escaped capture by the larger galaxies. It is possible that we may be able to detect such objects directly in the multibeam survey, and measure their dark matter contents dynamically. I find this an exciting prospect. If these small primordial objects are indeed present in the Scl group, then their $M_{\mathrm{HI}}$ values may be no larger than about $10^{5} M_{\odot}$, and integration times rather longer than 5 minutes will be needed to detect them.

A preliminary sparse survey of the Scl group, by Haynes \& Roberts (1979), did not detect most of Côté's dwarfs (which have $\mathrm{H}$ I masses $\sim 10^{8} M_{\odot}$ ), but did detect several $\mathrm{H}$ I clouds. The authors argued that these clouds may be associated with the Magellanic Stream rather than with the Sculptor group itself. We note, however, that the cloud velocities do appear to be generally somewhat higher than the Magellanic Stream velocities in this direction.

\section{Some Comments on Dark Matter}

Kormendy (1990) has shown how the characteristic central densities of the dark halos of galaxies scale with their absolute magnitudes. For different kinds of galaxies, the central densities of the dark halos are estimated by different methods (rotation curves for the larger spirals and irregulars, hydrostatic equilibrium of the $\mathrm{HI}$ component for the smallest irregulars, and stellar dynamics for the nearby dwarf spheroidal galaxies). Despite the diversity of methods, a recent study of this scaling law (Kormendy \& Freeman 1997) shows that the dynamically derived halo densities are tightly correlated with the absolute magnitudes. The halo densities range from about $10^{-3} M_{\odot} \mathrm{pc}^{-3}$ for the brightest spirals with $M_{\mathrm{B}} \approx-22$ to a very high value of about $1 M_{\odot} \mathrm{pc}^{-3}$ for the faintest dwarfs with $M_{\mathrm{B}} \approx-8$. For comparison, the total density of the galactic disk near the Sun is about $0.1 M_{\odot} \mathrm{pc}^{-3}$. What is the reason for these very high densities ( 1 $M_{\odot} \mathrm{pc}^{-3}$ ) of the dark matter components in the faintest dwarfs? Maybe the dark matter is dissipative, as Pfenniger, Combes \& Martinet (1994) have suggested. If it is not dissipative, then maybe we should look to the early Universe just after recombination, at a time when the density of the Universe itself was $\sim 1 M_{\odot} \mathrm{pc}^{-3}$. The smallest halos, with masses $\sim 10^{7} M_{\odot}$, may then be small bound objects from this early epoch. The lower densities of the larger halos may then reflect the processes of aggregation and tidal disruption by which they were built up.

In this context, I should mention the recent results of the MACHO survey (Alcock et al. 1996). This survey has now detected 8 secure microlensing events towards the LMC, and the properties of these events are consistent with a massive galactic dark halo for which a fraction of about 0.6 by mass is made of compact objects with individual masses of about $0.5 M_{\odot}$. These numbers still have significant uncertainties, and both numbers are likely to increase as the survey proceeds (because more massive lensing objects can be detected as the photometric time series get longer). If these numbers turn out to be correct, then what could these objects be? They are unlikely to be normal stars, because very faint stellar photometry in the Hubble Deep Field (e.g. Elson, Santiago \& Gilmore 1996) indicates that less than $20 \%$ of the halo dark matter can be in the form of low-mass luminous stars. Another possibility is that they are old white dwarfs: from the discussion of dwarf galaxies in the previous paragraph, these white dwarfs could be the stellar remnants from a very active pregalactic burst of star formation not long after recombination. This possibility brings with it some unresolved problems of chemical enrichment associated with the mass loss from the progenitors of these white dwarfs as they evolved. Another possibility is that they are primordial black holes, formed in the first $\sim 10^{-5} \mathrm{~s}$ of the Universe. Primordial black holes could have 
a very wide range of masses, however, and it is not clear why their masses should be similar to those of normal stars.

\section{Conclusion}

The multibeam H I Parkes all-sky survey will provide a new HI view of the sky, unbiased by optical selection. It will no doubt detect large numbers of galaxies with normal optical counterparts. But we can also expect it to discover other classes of objects. These include (1) intergalactic HI clouds; (2) very low-surface-brightness gas-rich galaxies (giants and dwarfs), which may come from the high- $\lambda$ tail of the distribution of protogalaxies; and (3) small $\mathrm{HI}$ clouds in nearby groups of galaxies, which may represent the primordial building blocks of galaxies.

Alcock, C., et al. 1996, ApJ, in press

Briggs, F. 1990, AJ, 100, 999
Côté, S. 1995, PhD Thesis, Australian National University Elson, R., Santiago, B., \& Gilmore, G. 1996, New Astronomy, in press

English, J. 1994, PhD Thesis, Australian National University Fall, S. M. 1985, in The Milky Way Galaxy, ed H. van Woerden et al. (Dordrecht: Reidel), p. 603

Freeman, K. C. 1987, Ann. Rev. Astron. Astrophys., 25, 603

Freeman, K. C. 1996, in Unsolved Problems of the Milky Way, ed. L. Blitz and P. Teuben (Dordrecht: Kluwer), p. 645

Giovanelli, R., \& Haynes, M. 1989, ApJ, 346, L5

Giovanelli, R., et al. 1995, AJ, 109, 1451

Haynes, M., \& Roberts, M. 1979, ApJ, 227, 767

Kennicutt, R. 1989, ApJ, 344, 685

Kormendy, J. 1990, in Evolution of the Universe of Galaxies (San Francisco: ASP), p. 33

Kormendy, J., \& Freeman, K. C. 1997, in preparation

Pfenniger, D., Combes, F., \& Martinet, L. 1994, A\&A, 285, 79

Schneider, S. 1989, ApJ, 343, 94

Zurek, W., et al. 1988, ApJ, 330, 519 